\title{
Novel Approaches in Designing Natural/Synthetic Materials for Environmental Applications
}

\author{
George Z. Kyzas, ${ }^{1,2}$ Saeid Azizian, ${ }^{3}$ and Margaritis Kostoglou ${ }^{1}$ \\ ${ }^{1}$ Division of Chemical Technology, Department of Chemistry, Aristotle University of Thessaloniki, 54124 Thessaloniki, Greece \\ ${ }^{2}$ Department of Petroleum and Natural Gas Technology, Technological Educational Institute of Kavala, 65404 Kavala, Greece \\ ${ }^{3}$ Department of Physical Chemistry, Bu-Ali Sina University, Hamedan 64174, Iran
}

Correspondence should be addressed to George Z. Kyzas; georgekyzas@gmail.com

Received 25 September 2014; Accepted 25 September 2014

Copyright (C) 2015 George Z. Kyzas et al. This is an open access article distributed under the Creative Commons Attribution License, which permits unrestricted use, distribution, and reproduction in any medium, provided the original work is properly cited.

This is one of a series of special issues published in this journal, focusing on the latest advances of designing natural or synthetic materials for using them in environmental applications.

The designing of materials is one of the most developing sectors of environmental science. Novel materials can be synthesized having the ultimate goal of an environmental application. In this field many parameters play an important role as biodegradability, reuse/regeneration, cost, and environmental-friendly disposal. Other standards will be also taken into account as conditions of synthesis, possible use in columns, and so forth. This issue includes novel approaches in designing either natural or synthetic materials which will have direct application to environmental targets (dyes, heavy metals/ions, phenols, pesticides/insecticides, nuclear pollutants, etc.). The materials described can be used either for gas or liquid use (i.e., adsorption). Extra attention and priority will be given to real samples of environmental pollutants (industrial effluents). Various types of works were selected to be published with wide topics. The technique of molecular imprinting was introduced by G. Z. Kyzas et al. for selectively binding of high-added value metals. Yang et al. reported material developments regarding geotechnical engineering, while Y. Zhang et al. extensively describe the synthesis and characterization of star-shaped block copolymer sPCL-bPEG-GA. S.-W. Myoung et al. investigated the thermal barrier coatings in cyclic thermal exposure as well as the effect of plasma pretreatment on their thermal durability, while some other related topics have been also published, such as the unstable temperature distribution in friction stir welding (by S. Aziz et al.), the designing and simulated characteristics of nanosized InSb based heterostructure devices (by T. D. Subash et al.), and the synthesis of raw materials originated from industrial effluents for bioremediation and phytomining (by S. B. Karman et al.).

Under the direction of the editorial team, we showcase advances of organic and inorganic based smart materials and their applications in areas of specific interest such as energy, environment, and health.

\section{Acknowledgments}

We gratefully thank all contributing authors for sharing their research outputs and time, thus enabling the successful accomplishment of this issue.

George Z. Kyzas

Saeid Azizian

Margaritis Kostoglou 

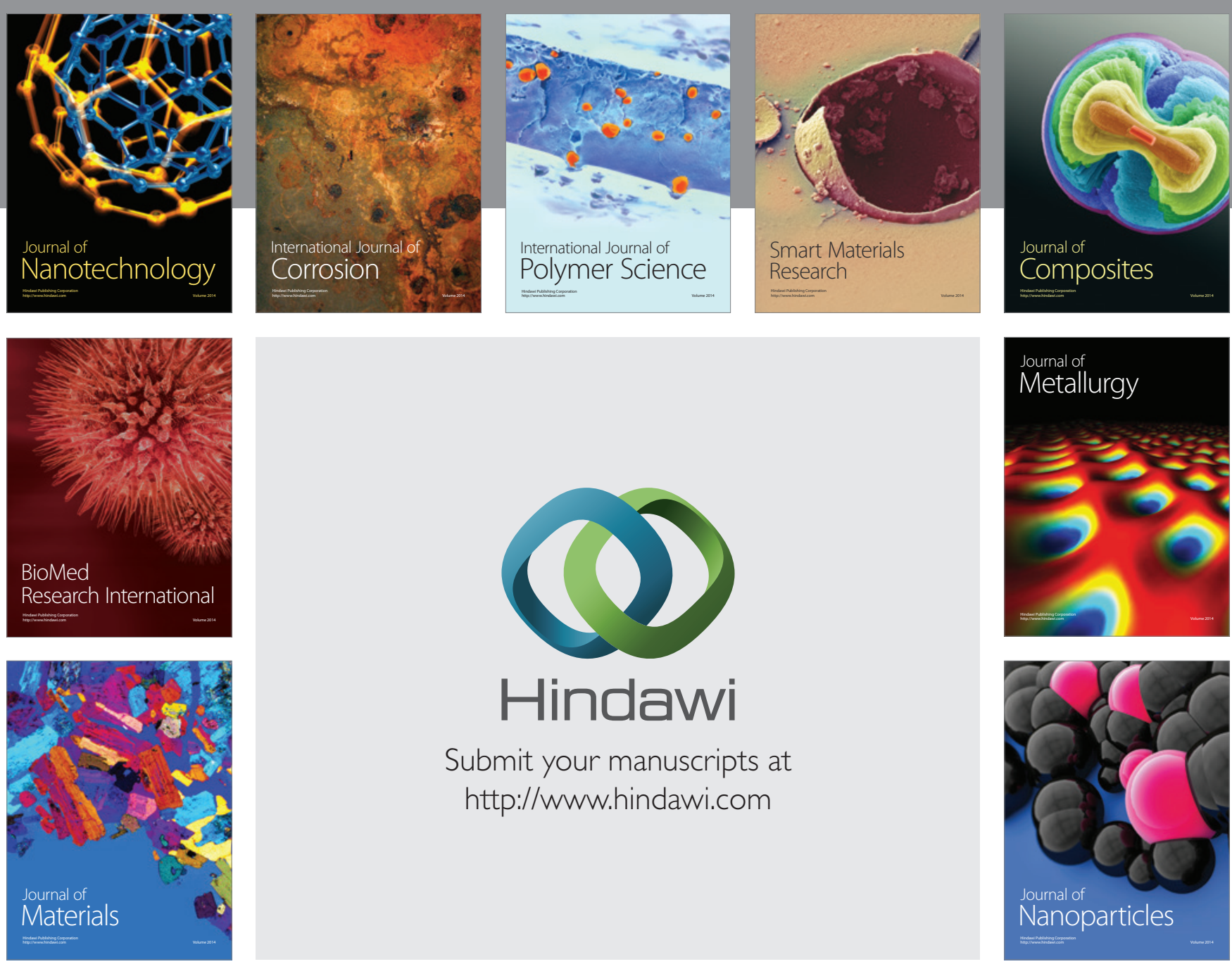

Submit your manuscripts at http://www.hindawi.com
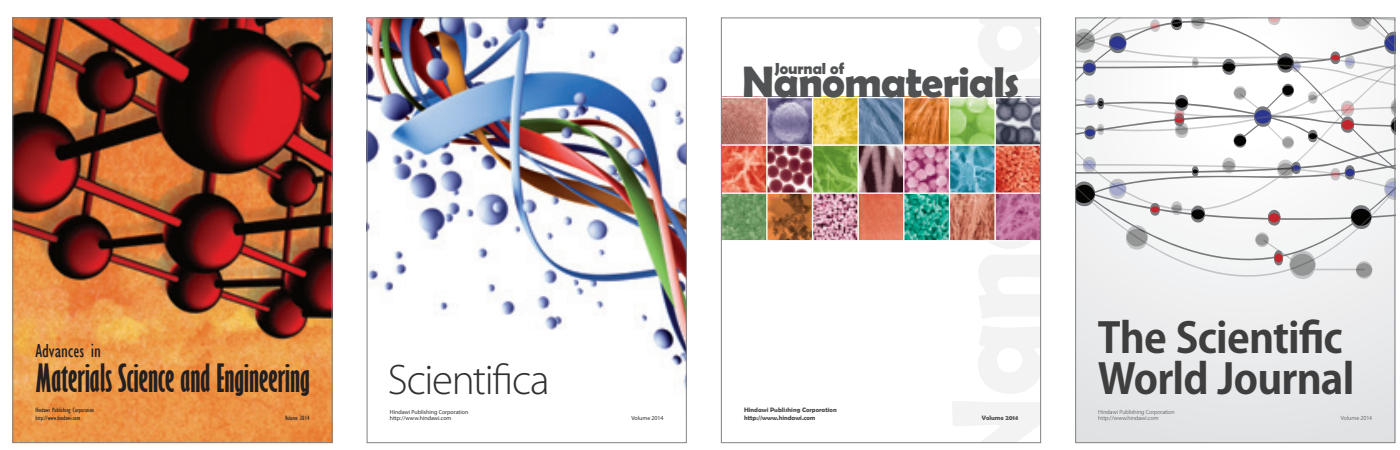

\section{The Scientific World Journal}
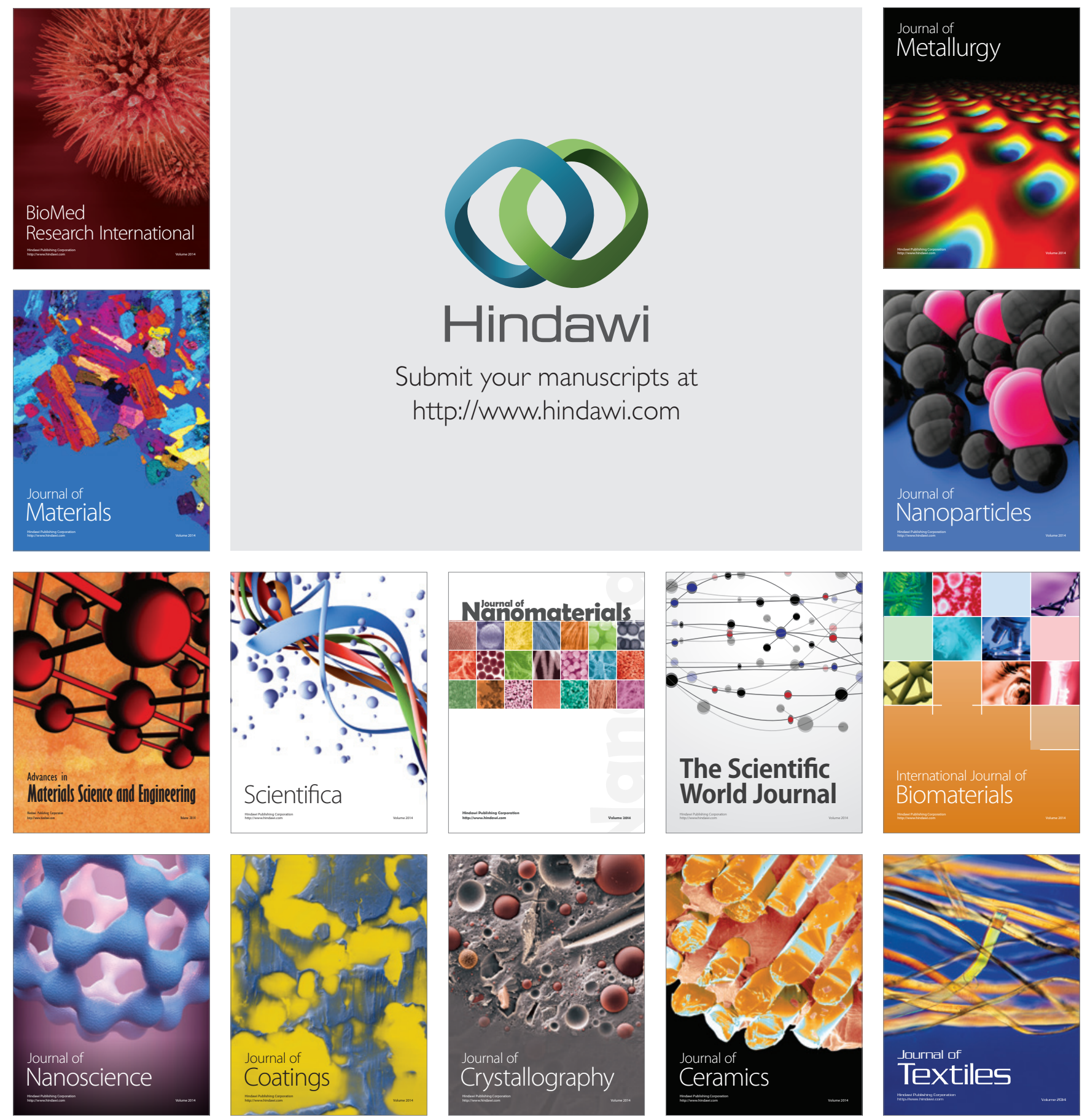